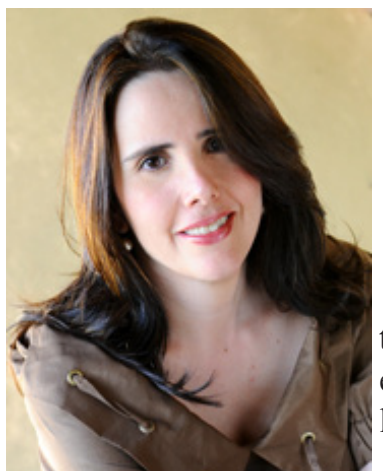

\title{
Diversity and Mental Health: potential for EXCLUSION OR DIALOGUE?
}

\section{Carla Aparecida Arena Ventura}

Diversity is a grouping under which multiple aspects are gathered together that are different from each other, which can generate social stimuli with extremely positive results, when understood as a possibility for understanding and learning with the other. However, it can also produce painful and negative results,

particularly in situations where difference is associated with the unknown, which individuals distance themselves from and exclude. In these cases, barriers are raised that legitimize demarcations, generating profound impacts on the identity of the individuals.

Mental health encompasses and combines different aspects of diversity, such as the social, values, and information $^{(1)}$. For the $\mathrm{WHO}^{(2)}$, the concept of mental health is analogous to that of health, associating it with complex phenomena, influenced by different cultures and by subjectivity, since individuals with mental disorders generally have a multifaceted set of clinical and social needs.

In this context, diversity in mental health has historically highlighted the individual and collective difficulties of facing "insanity", with devastating consequences for those with mental disorders, by means of consolidating the culture of asylums and banalizing the reification of these human beings, completely deprived of their individuality.

Furthermore, the movements for reforming and consolidating human rights, as the rights of every human person, paved the way for dialogue as an instrument to work with these challenges and to generate new perspectives and forms of action. In this new light, various modes of understanding and relating to these individuals are sought out, rather than reducing them to their diseases. After years of exclusion, the possibility of dialogue is envisioned in the context of diversity in mental health, with the production of evidence and the implementation of practices that are accepting and are not intimidated by its complexity, generating guided technical actions to affirm difference and deny its inequalities.

This edition of the Mental Health, Alcohol, and Drugs Magazine reflects the diversity intrinsic to mental health, combining articles with distinct topics: the meaning attributed by workers at a CAPS AD (Psychosocial Care Center for Alcohol and Drugs) to caring for crack users; interactions of drugs administered at a Emergency Psychiatric Service at a general hospital during the first hours of care; the pattern of consumption of alcoholic beverages in young people during their education; neonatal repercussions resulting from exposure to crack during gestation; adherence to medication therapy by CAPS users with mood disorders and schizophrenia; depression in pregnant women towards the end of gestation; and two integrative reviews: the first on the nursing consultation in mental health in primary care, and the second on the applicability of the nursing process in psychiatric nursing care. In addition to a number of topics on mental health, alcohol, and drugs, the articles originate from different regions of Brazil and one was prepared in Lisbon, Portugal.

In short, the diversity in research in mental health and on alcohol and drugs becomes inclusive and sensitive to the needs of the more vulnerable groups, accelerating and reinforcing advances, offering new forms of looking at "old" and recurring problems. This plurality allows for the area of knowledge to evolve by allowing research to fill in the gaps and consolidate an agenda that legitimately seeks to answer real social needs. In this regard, there is still the important challenge of recognizing diversity in mental health and seeking instruments, by means of education and research, to work with it, reducing the stigma and the discrimination of individuals with mental disorders and users of alcohol and other drugs. 


\section{References}

1. Worman D. Is there a business case for diversity? Personn Today. May 2005:27-8.

2. World Health Organization. Mental health: strengthening mental health promotion. [Internet]. Fact Sheet N. 220,2007 [Acesso 2 março 2016]. Disponível em: www.who.int/mediacentre/factsheets/fs220/en/

\section{Carla Aparecida Arena Ventura}

Associate Editor of the SMAD, Revista Eletrônica Saúde Mental Álcool e Drogas, Full Professor of the University of São Paulo at Ribeirão Preto College of Nursing, PAHO/WHO Collaborating Centre for Nursing Research Development, Brazil, e-mail: caaventu@eerp.usp.br. 